\title{
Protector bucal por laminado a presión en paciente deportista
}

\section{Pressure lamination technique mouthguard for athlete patient}

\author{
Camila Corral ${ }^{1,2,3^{*}}$, Montserrat Mercado ${ }^{1}$, André Latapiat $^{1}$, Andrea Veliz $^{1}$, \\ Mauricio Garrido ${ }^{1}$, Osmir Batista de Oliveira $\mathrm{Jr}^{3}$
}

1. Clínica de Traumatismo Dentoalveolar del Niño y del Adulto, Facultad de Odontología, Universidad de Chile, Chile.

2. Departamento de Odontología Restauradora, Facultad de Odontología, Universidad de Chile, Chile. 3. Departmento de Odontología Restauradora, Facultad de Odontología, Universidade Estadual Paulista, UNESP -Araraquara, Brasil

${ }^{*}$ Correspondencia autor: Camila Corral Núñez | Clínica de Traumatismo Dentoalveolar del Niño y del Adulto, Facultad de Odontología, Universidad de Chile, Chile. Sergio Livingstone Pohlhammer 943, Independencia, Santiago, Chile | e-mail: camila.corral@u.uchile.cl. Trabajo recibido el 13/03/2017. Aprobado para su publicación el 28/08/2017

\section{RESUMEN}

El traumatismo dentoalveolar incluye un conjunto de lesiones que afectan a los dientes y/o estructuras de soporte a consecuencia de un impacto violento directo o indirecto. Debido a que por lo general comprometen el sector anterior generan cambios físicos, fonéticos, psicológicos y dietarios, que pueden afectar significativamente la calidad de vida de pacientes afectados por este traumatismo.

Los protectores bucales son considerados la principal medida para minimizar y/o evitar estos traumatismos. El presente artículo describe el tratamiento y procedimientos para realizar un protector bucal por laminado a presión en un paciente deportista con historia previa de traumatismo dentoalveolar en incisivos centrales superiores

\section{PALABRAS CLAVE}

Protector Bucal; Traumatismo Dentoalveolar; Prevención.

Rev. Clin. Periodoncia Implantol. Rehabil. Oral Vol. 10(3); 166-168, 2017.

\section{ABSTRACT}

Traumatic dental injuries involve lesions that affect teeth and/or adjacent supporting structures as a consequence of direct or indirect violent impact. Due to As it affects the anterior teeth, in most of the cases, it provokes changes in the physical appearance, altering phonetics, psychology and the diet of the affected subject. These changes may significantly alter the quality of life of patients affected by this type of trauma.

Mouthguards are considered the primary appliance for minimizing and/or avoiding dental trauma. The present article describes the treatment and procedures to perform a mouthguard by pressure lamination technique in a patient that who practices sports and with a history of trauma affecting the upper central incisors.

KEYWORDS

Mouthguard; Traumatic Dental Injuries; Prevention.

Rev. Clin. Periodoncia Implantol. Rehabil. Oral Vol. 10(3); 166-168, 2017.

\section{INTRODUCCIÓN}

El traumatismo dentoalveolar (TDA) es considerado una patología irreversible, que incluye un conjunto de lesiones que comprometen a los dientes y/o sus estructuras de soporte, a consecuencia de un impacto violento directo o indirecto(1). Este constituye un serio problema de salud pública(2), que presenta una alta prevalencia, a pesar de que la región oral comprende sólo un $1 \%$ del área total del cuerpo ${ }^{(3)}$. Los resultados epidemiológicos de la mayoría de los países, muestran que aproximadamente un tercio de los preescolares, un cuarto de los escolares y un tercio de los adultos ha sufrido un TDA a lo largo de su vida ${ }^{(2)}$.

Debido a que la mayoría de los TDA involucran dientes del sector anterior, muchas veces estos tienen diversas consecuencias, entre ellas restricciones dietarias, cambios en la apariencia física, dificultades fonéticas e impactos psicológicos que pueden afectar la calidad de vida del individuo afectado ${ }^{(4,5)}$. Estas lesiones son comunes no sólo en deportes de alto riesgo de contacto (como box, artes marciales mixtas, hockey o rugby), si no también en otros deportes en que el riesgo de contacto no es tan patente (como skateboard, snowboard, montain bike básquetbol, gimnasia y patinaje)(6).

Los protectores bucales (PBs) han sido considerados como el principal medio de protección para minimizar las heridas orales en actividades deportivas ${ }^{(6)}$. Los PBs reducen la severidad de la injuria y previenen laceraciones de tejidos blandos, el desplazamiento, fractura o avulsión de dientes, fracturas de proceso alveolar, entre otros.
El siguiente reporte de caso muestra el procedimiento de confección de un protector bucal con técnica de laminado por presión en un paciente deportista joven, posterior a tratamiento por traumatismo dentoalveolar.

\section{REPORTE DE CASO}

Paciente sano, sexo masculino, 11 años, que acude a la Clínica de Traumatismo Dentoalveolar del Niño y del Adulto, Facultad de Odontología, Universidad de Chile para realizar tratamiento de incisivos centrales superiores (11 y 21 ) con fractura coronaria complicada, producto de caída al bajar una bicicleta de camioneta. El paciente practica Bicicross-BMX, compitiendo a nivel internacional como seleccionado nacional. El tratamiento del paciente incluyó terapia de regeneración pulpar en incisivos centrales superiores y reconstrucción estética con resina compuesta Miris ${ }^{\circledR} 2$ (Coltene/Whaledent). Posterior a la evaluación de riesgo del paciente, en que se determinó un alto riesgo de TDA debido al deporte que practica, se procedió a la fabricación de un PB individualizado con técnica de laminado.

Para la fabricación del PB individualizado se realizó registro de arcada superior e inferior con impresión de alginato Jeltrate ${ }^{\circledR}$ Dustless (Dentsply) incluyendo el fondo de vestíbulo, en cubetas metálicas tipo Rimlock, posteriormente se realizó registro de mordida con silicona pesada. Las impresiones fueron vaciadas en yeso Piedra (Yeso Piedra Elite ${ }^{\circledR}$ Blanco), para la obtención de modelos.

Los modelos y registro de mordida fueron enviadas a laboratorio dental, con las indicaciones del tipo de PB requerido, en este caso de 
categoría Heavy, Playsafe ${ }^{\circledR}$ Competition Mouthguards y la indicación de diseño de colores que el paciente seleccionó. El PB de categoría Heavy corresponde a un plano con doble lámina de polietileno acetato de polivinilo (EVA), con tres bandas de transmisión de energía en caso de impacto, que se realiza con técnica de laminado a presión.

El PB realizado se observa en Figura 1. Este se diseñó en forma de herradura, conteniendo los dientes de la arcada superior con extensión vestibular hasta $2 \mathrm{~mm}$ bajo el fondo de vestíbulo y por palatino extendiéndose $3 \mathrm{~mm}$ desde el margen gingival. Además, presenta indentaciones de premolares y molares inferiores en la cara externa del PB. Este PB presenta una banda radiopaca, que facilita su ubicación en caso de accidentes.
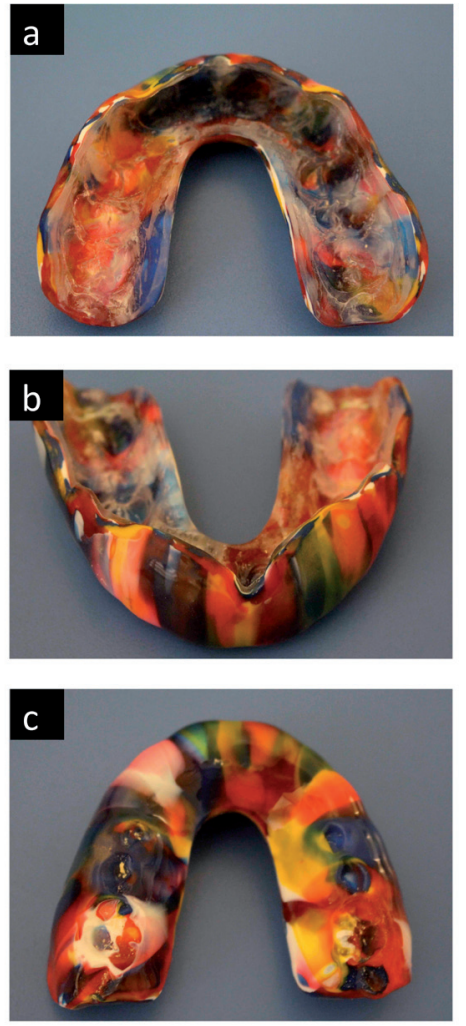

Figura 1. Protector bucal a) cara interna, b) cara externa, c) cara oclusal mostrando indentaciones.

En la siguiente sesión se realizó la prueba del PB en la cavidad oral del paciente (Figura 2) revisando la correcta retención, adaptación a los tejidos y comodidad. Posteriormente se le entregaron al paciente las indicaciones de uso, cuidado e higiene:

- La limpieza del PB se debe realizar sólo con agua tibia y jabón.

- Antes de utilizar el PB, este debe ser enjuagado.

- Evitar la exposición del PB a temperaturas muy altas o muy bajas.

- No compartir el uso del PB.

- Cuando el PB no está siendo utilizado, se recomienda mantenerlo en estuche plástico limpio y seco.

- Si el PB se siente seco, se puede aplicar fina capa de vaselina.

Finalmente, se le recomendó al paciente utilizar el PB en toda ocasión en que practique deporte, en caso de molestias con su uso solicitar atención y acudir a control cada 6 meses para evaluar el PB.

\section{DISCUSIÓN}

Varios estudios han demostrado el valor protector de los PBs en reducir lesiones a dientes y tejidos adyacentes producto de accidentes relacionados a actividades deportivas ${ }^{(7,8)}$. Los PBs ofrecen protección absorbiendo y disipando la energía de impactos que de no estar presentes serían transferidos directamente a los tejidos dentales. Más aún, existe evidencia que reporta que los PBs son efectivos también en proteger contra concusión cerebral e injurias a columna cervical y articulación temporo-mandibular ${ }^{(9)}$.

Actualmente, de acuerdo a la Academy for Sports Dentistry (ASD) existen tres tipos de PBs disponibles ${ }^{(10)}$ :
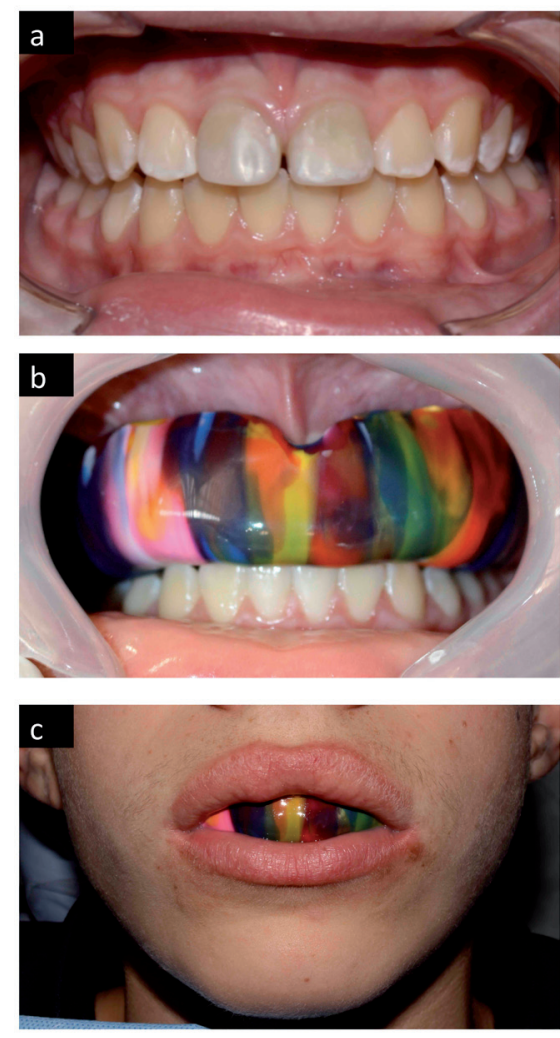

Figura 2. (a) Paciente con incisivos centrales superiores (11 y 21) restaurados post traumatismo dentoalveolar, (b) y (c) protector bucal en posición.

(i) Tipo 1: Prefabricados o no adaptables, "ready to use", cubeta preformada sin capacidad para adaptarse ${ }^{(7)}$. Este tipo de PB es de bajo costo, en general de polivinilcloruro, poliuretano, o un copolímero de vinil acetato o etileno(11). Se vende en tamaños limitados y tiene poca retención y adaptación a los tejidos ${ }^{(11)}$. Para ejercer su acción preventiva requiere que el sujeto esté ocluyendo, por tanto, interfiere con la respiración y la capacidad para hablar. Por estas razones es considerado como el de menor protección.

(ii) Tipo 2: Adaptables en boca(7). El más común de ellos se conoce como "boil-and-bite", que es una cubeta estándar de material termoplástico que se puede ajustar después de mantenerlo en agua caliente $^{(11)}$. Este ajuste se realiza con los dedos y presión de la lengua y mejillas, lo podría realizar el paciente, pero se ha recomendado sean adaptados por un dentista(12). Se discute su eficacia, ya que puede quedar muy delgado en algunas zonas, y no ser capaz de amortiguar los impactos de manera efectiva ${ }^{(11)}$.

(iii) Tipo 3: Individualizados, se realizan sobre modelos obtenidos a través de una impresión de alginato, por lo general se confeccionan en EVA (polietileno acetato de polivinilo)(11,12). De costo más elevado y por lo general se realizan en laboratorios dentales. Estos PBs son diseñados individualmente y ajustados a la anatomía bucal del modelo por dos técnicas:

a. Técnica de formación al vacío o por estampado: Requiere de máquina de vacío (estampadora), en que una única lámina termoplástica, se calienta y ajusta al modelo por succión (equivalente a aproximadamente una atmosfera de presión $)^{(6)}$. Se obtienen protectores de una capa, con variables grado de grosor ${ }^{(12,13)}$.

b. Técnica de laminado a presión: Se utiliza una máquina de presiónlaminado, la combinación de calor y presión (hasta 10 atmósferas) permite adaptar la lámina al modelo(6). Capas adicionales se pueden laminar (usualmente 2 o 3 capas de EVA) hasta lograr el espesor necesario, permite añadir o aumentar el grosor en zonas específicas, presenta un ajuste más preciso que la técnica por vacío(6,12).

A pesar del mayor costo económico de los PBs individualizados, la literatura demuestra que presentan numerosas ventajas frente a las otras alternativas incluyendo mayor protección, mejor retención, confort y un deterioro más lento ${ }^{(12)}$. Sin embargo, el determinar qué técnica es más óptima para construir PBs individualizados permanece siendo un tópico de interés profesional( ${ }^{(6)}$. Se han sugerido tres áreas para mejorar los 
PBs: mejorar materiales (que absorban impactos repetitivos y transfieran menos energía a los dientes), mejorar diseño del PBs y desarrollar un efectivo y objetivo test para evaluar $\mathrm{PBs}^{(11)}$

A pesar del importante rol de los PBs en prevenir lesiones, es sorprendente lo poco usado que es. Se ha demostrado que existe una tendencia en el público general en ignorar mensajes de seguridad y salud, sin importar cuan contundente sea la evidencia, ya que individuos tienden a creer que los riesgos son solo aplicables a otros ${ }^{(14)}$. El bajo uso de PBs se debe en parte a la falta de regulación y educación de deportistas en este ámbito ${ }^{(6)}$. Sin embargo, también se ha reportado un bajo uso en niños que tienen PBs con causas como "no me gusta usarlo", "es incómodo", "se me olvida ocuparlo" y "soy el único niño que usa PB dentro de mis amigos"(15). Por otra parte, los padres en un alto porcentaje han reportado no saber de la existencia de PBs, y que dentistas no les han ofrecido este tratamiento en el pasado(15). Es por ello, que es importante la educación a deportistas, padres y entrenadores por parte de dentistas, aconsejando el uso de PBs. Teniendo siempre en mente que la clave del mensaje es que el mejor PBs es aquel que se usa. Mientras que el PBs individualizado se considera como la mejor protección, otros PBs también pueden ser efectivos si se usan apropiadamente.

\section{CONCLUSIÓN}

De acuerdo a la evidencia disponible, se sugiere el uso de PBs en toda actividad deportiva o recreativa que implique algún riesgo de lesión en los tejidos dentoalveolares. Diversos estudios reportan que los protectores bucales individualizados otorgan mejor protección, retención y confort, por lo tanto, debieran ser recomendados.

\section{Bibliografía}

1. Andreasen JO, Lauridsen E, Christensen SS. Development of an interactive dental trauma guide. Pediatr Dent. 2009;31(2):133-6.

2. Glendor U. Epidemiology of traumatic dental injuries--a 12 year review of the literature. Dent Traumatol. 2008;24(6):603-1.

3. Petersson EE, Andersson L, Sorensen S. Traumatic oral vs non-oral injuries. Swed Dent J. 1997:21(1-2):55-68.

4. Thelen DS, Bardsen A. Traumatic dental injuries in an urban adolescent population in Tirana, Albania. Dent Traumatol. 2010;26(5):376-82.

5. Soriano EP, Caldas Ade F, Jr., Diniz De Carvalho MV, Amorim Filho Hde A. Prevalence and risk factors related to traumatic dental injuries in Brazilian schoolchildren. Dent Traumatol. 2007:23(4):232-40.

6. ADA. Using mouthguards to reduce the incidence and severity of sports-related oral injuries. JADA. 2006;137(12):1712-20.

7. Newsome PR, Tran DC, Cooke MS. The role of the mouthguard in the prevention of sports-related dental injuries: a review. Int J Paediatr Dent. 2001;11(6):396-404.

8. Knapik JJ, Marshall SW, Lee RB, Darakjy SS, Jones SB, Mitchener TA, et al. Mouthguards in sport activities : history, physical properties and injury prevention effectiveness. Sports Med. 2007;37(2):117-44.
9. Tanaka Y, Tsugawa T, Maeda Y. Effect of mouthguards on impact to the craniomandibular complex. Dent Traumatol. 2016;33(1):51-56.

10. Maeda Y, Kumamoto D, Yagi K, Ikebe K. Effectiveness and fabrication of mouthguards. Dent Traumatol. 2009;25(6):556-64.

11. Patrick DG, van Noort R, Found MS. Scale of protection and the various types of sports mouthguard. Br J Sports Med. 2005;39(5):278-81.

12. Guinot Jimeno F, Torrens Gras R, Armengol Olivares A, Cuadros Fernández $\mathrm{C}$, Lorente Rodríguez A. Protector bucal individualizado mediante la técnica de formación al vacío. A propósito de un caso. Odontol Pediátr. 2014;22(3):195-208.

13. Mizuhashi F, Koide K, Takahashi M. Thickness and fit of mouthguards according to a vacuum-forming process. Dent Traumatol. 2013;29(4):307-12.

14. Newsom,D, Turk,JS, Kruckeberg,D. This is PR: The realities of public relations, 6th edition. London: Wadsworth Pub. Co; 1996.

15. Matalon V, Brin I, Moskovitz M, Ram D. Compliance of children and youngsters in the use of mouthguards. Dent Traumatol. 2008;24(4):462-7. 